\title{
TRASTORNOS DEL SUEÑO A CONSECUENCIA DE LA PANDEMIA POR COVID-19
}

\author{
Oscar Medina-Ortiz(1),a, Franlet Araque-Castellanos (101,b, Luis Carlos Ruiz-Domínguez(101,c, \\ Manuel Riaño-Garzón(101,d, Valmore Bermudez(1),e \\ ${ }^{1}$ Facultad de Ciencias Jurídicas y Sociales, Universidad Simón Bolívar, Cúcuta, Colombia. \\ a Médico especialista en Psiquiatría y Medicina del sueño, doctor en Neurociencias; ${ }^{\mathrm{b}}$ psicóloga, magíster en Estudios \\ Culturales; ${ }^{\mathrm{c}}$ psicólogo, magíster en Neuropsicología y Educación; ${ }^{\mathrm{d}}$ psicólogo, magíster en Psicología Clínica; ${ }^{\mathrm{e}}$ médico \\ cirujano, doctor en Ciencias Médicas.
}

\section{RESUMEN}

Las complicaciones respiratorias por la COVID-19 pueden llevar a la muerte, por lo que, dentro de las políticas de protección para evitar los contagios masivos, se han sugerido estrategias de cuarentena y confinamiento en muchos países, que han originado alteraciones en la salud mental y el sueño. A través de la siguiente revisión narrativa se pretende sistematizar los hallazgos más significativos en cuanto a la presencia de insomnio en pacientes hospitalizados con COVID-19, y de personas sanas que han estado sometidas a confinamiento como medida preventiva. Las condiciones propias de la enfermedad han hecho que los pacientes desarrollen insomnio, lo que puede empeorar su estado de salud y alterar su sistema inmunológico. Para las personas sanas en cuarentena los cambios en el estilo de vida, el miedo a contraer la enfermedad, la edad joven, el sexo femenino, los antecedentes de enfermedades mentales y una menor capacidad de afrontamiento al estrés parecen ser factores de riesgo para el insomnio. Al igual que la implementación de medidas epidemiológicas de cuidado y prevención contra el COVID-19, se debe tener en consideración promover la higiene del sueño como una estrategia de afrontamiento integral contra esta pandemia.

Palabras clave: Insomnio; COVID-19; Pandemia; Cuarentena (Fuente: DeCS BIREME).

\section{SLEEP DISORDERS AS A RESULT OF THE COVID-19 PANDEMIC}

\begin{abstract}
Respiratory complications from COVID-19 can lead to death. For this reason, public health measures to curb the spread of the disease such as quarantine and other confinement strategies have been proposed in several countries, resulting in mental health and sleep disorders. We carried out a narrative review to systematize the most significant findings regarding insomnia in hospitalized patients with COVID-19, and in healthy persons who have been under confinement as a preventive measure. COVID-19-related conditions have caused insomnia in patients, which can alter the immune system and have a negative effect on health. For healthy people in quarantine, lifestyle changes, fear of becoming infected, young age, female gender, history of mental illness and reduced ability to cope with stress appear to be risk factors for insomnia. As well as the implementation of epidemiological and preventive measures, sleep hygiene should be promoted as a comprehensive coping strategy against the COVID-19 pandemic.
\end{abstract}

Citar como: Medina-Ortiz O, Araque-Castellanos F, RuizDomínguez LC, Riaño-Garzón M, Bermudez V. Trastornos del sueño a consecuencia de la pandemia por COVID-19. Rev Peru Med Exp Salud Publica. 2020;37(4):755-61. doi: https://doi.org/10.17843/ rpmesp.2020.374.6360

Correspondencia: Oscar Medina Ortiz; dr.oscarmedina@gmail.com

Recibido: $19 / 08 / 2020$ Aprobado: $28 / 10 / 2020$ En línea: $11 / 11 / 2020$
Keywords: Insomnia; COVID-19; Pandemic; Quarantine (Source: MeSH NLM).

\section{INTRODUCCIÓN}

La COVID-19 fue declarada como pandemia por la OMS el 11 de marzo de $2020^{(1)}$, y tuvo su inicio como un nuevo tipo de coronavirus en la ciudad de Wuham, provincia de Hubei, China, el 12 de diciembre de $2019^{(2)}$. Cuando una persona se infecta, los primeros síntomas pueden aparecer entre el quinto y sexto día con predominio de fiebre, tos y fatiga, pudiendo presentar también cefalea y diarrea ${ }^{(3,4)}$. Aun cuando la mayoría de las personas presentan síntomas leves, un pequeño grupo puede complicarse con síndrome de insuficiencia respiratoria aguda, insuficiencia renal aguda y trastornos cardiovasculares, que pueden poner en riesgo su vida ${ }^{(5)}$. 
Para prevenir la transmisión de la infección entre los ciudadanos, los gobiernos de diferentes países han propuesto un periodo variable de aislamiento y cuarentena como medida de salud pública ${ }^{(6)}$. Pero, si bien esta estrategia se ha diseñado con la intención de cuidar la salud física de las personas, es necesario considerar que después de cierto tiempo, se puede ver afectada la salud mental de quienes son sometidos a este tipo de restricciones, que incluyen, entre otras, confinamiento en sus hogares y aislamiento social ${ }^{(7)}$.

Una revisión llevada a cabo por Hossain M. et al. describe nueve estudios publicados entre 2009 y 2020 donde se muestran las implicaciones del aislamiento preventivo en la salud mental, destacando en primer lugar la presencia de síntomas relacionados con ansiedad y depresión ${ }^{(8)}$. El dormir no escapa a estas circunstancias, ya que el miedo juega un factor determinante en la modificación de los patrones normales de sueño ${ }^{(9,10)}$. Sin embargo, no solo las personas en cuarentena pueden presentar este tipo de alteraciones, sino también los pacientes que sufren la enfermedad, siendo el insomnio un síntoma relevante en quienes permanecen hospitalizados por la COVID-19 ${ }^{(11)}$.

Según la clasificación internacional de los trastornos del sueño (ICSD-3), publicada por la Academia Americana de Medicina del Sueño (AASM) en 2014, el insomnio se define como una dificultad persistente para iniciar el sueño con insatisfacción en su duración, consolidación o calidad, que ocurre cuando las circunstancias y oportunidades para dormir están dadas, y se acompaña al día siguiente de malestar o deterioro en las funciones sociales, laborales, educativas, académicas, conductuales o en otras áreas importantes de la conducta humana ${ }^{(12)}$. La prevalencia del insomnio varía entre el 5 y el 50\% cuando se toman en consideración solo las características nocturnas (dificultad para iniciar o mantener el sueño), pero cuando se incluyen todos los factores mencionados anteriormente, que constituyen el síndrome clínico completo de insomnio crónico, la prevalencia puede bajar y ubicarse en un rango que oscila entre el 6 y el 10\% de la población general ${ }^{(13)}$.

El insomnio se clasifica principalmente en crónico y de corta duración. En el insomnio crónico, los síntomas deben estar presentes por lo menos tres veces a la semana durante un periodo de tres meses y puede deberse, entre otras cosas, a la presencia de una enfermedad médica crónica que produce dolor, malestar general o dificultad para respirar ${ }^{(14,15)}$. El insomnio de corta duración se diagnostica durante los primeros tres meses y puede ser debido a un evento o condición estresante en la vida de las personas, como pérdidas afectivas, discusiones familiares, muerte de un ser querido, y podría incluirse en este grupo la incertidumbre permanente ante el posible contagio de esta nueva enfermedad, exacerbado por la información emitida en los medios de comunicación y redes sociales ${ }^{(16)}$. En base a esta clasificación no sería tan fácil determinar en este momento cuál es el tipo de in- somnio que presenta un paciente durante el confinamiento por la COVID-19, debido a que la alteración del sueño podría deberse tan solo al temor de contagio; sin embargo, es necesario señalar que las intervenciones terapéuticas deben estar dirigidas a evitar que el insomnio de corta duración evolucione a crónico.

Se lleva a cabo esta revisión con el objetivo de mostrar los hallazgos de diferentes estudios sobre la prevalencia de insomnio en pacientes con COVID-19 y en población general sin COVID-19 sometidos a aislamiento y cuarentena, y la posible relación entre el insomnio y esta enfermedad.

\section{BÚSQUEDA Y SELECCIÓN DE ARTÍCULOS}

Se realizó una revisión narrativa a partir de la identificación de artículos científicos mediante una búsqueda rápida en las bases de datos PubMed, Science Direct, Scopus y Scielo, con las palabras insomnia, coronavirus, sleep, COVID-19, quarentine y isolation, en inglés y español (insomnio, coronavirus, sueño, COVID-19, cuarentena y aislamiento), se revisaron todos los artículos publicados hasta el mes de julio de 2020, sin límite de tiempo o edad y solo en humanos. Estas bases fueron escogidas por su alto nivel científico y alcance global.

En un principio se identificaron 45 estudios. Se excluyeron aquellos realizados en personal de salud, en los que el objetivo era solo la evaluación de aspectos psiquiátricos, donde solo se evaluaba calidad del sueño y en los que no se registró la prevalencia de insomnio. Se revisaron las referencias de los artículos incluidos para identificar literatura adicional. Al final se incluyeron 12 estudios (Tabla 1).

\section{INSOMNIO EN PACIENTES HOSPITALIZADOS POR COVID-19}

Wang Y et al. ${ }^{(17)}$ llevaron a cabo un estudio en marzo de 2020 para determinar cuáles eran los factores sociodemográficos asociados al insomnio en 484 pacientes mayores de 14 años, hospitalizados por la COVID-19 en el Hospital Tongji de Wuhan, en la provincia de Hubei, China. Se aplicó la versión china del Insomnia Severity Index (ISI-7) y el General Health Questionnaire (GHQ-12). El 42,8\% de los pacientes presentaban insomnio, el cual se asoció al sexo femenino ( $\mathrm{p}<0,001)$, estar casados $(\mathrm{p}=0,042)$, recibir información sobre la enfermedad a través de las redes sociales $(\mathrm{p}=0,010)$ $\mathrm{y}$ aquellos que presentaban fatiga severa $(\mathrm{p}<0,010)$. Este estudio mostró que cerca de la mitad de los pacientes hospitalizados por la COVID-19 presentaban insomnio. Uno de los hallazgos interesantes fue que quienes contaban con acceso a internet y a smartphones eran los jóvenes, y estos presentaban mayores puntajes para insomnio en el ISI-7, probablemente porque recibían más noticias sobre la evo- 
Tabla 1. Estudios sobre el insomnio en pacientes con la COVID-19 y en la población sana durante la cuarentena.

\begin{tabular}{|c|c|c|c|c|}
\hline Autores & País & Muestra & Resultados & Conclusiones \\
\hline $\begin{array}{l}\text { Yu Wang et al. } \\
(2020)^{(11)}\end{array}$ & China & $\begin{array}{l}484 \text { pacientes hospitalizados mayores de } 14 \\
\text { años. }\end{array}$ & $\begin{array}{l}\text { Más insomnio en las mujeres, en las personas } \\
\text { casadas, en quienes usan redes sociales y en } \\
\text { quienes se sienten más fatigados. }\end{array}$ & Insomnio $=42,8 \%$ \\
\hline $\begin{array}{l}\text { Zhang J. et al. } \\
(2020)^{(23)}\end{array}$ & China & $\begin{array}{l}135 \text { pacientes hospitalizados entre } 18 \text { y } 80 \\
\text { años. }\end{array}$ & $\begin{array}{l}\text { El grupo de malos durmientes presentó valores } \\
\text { absolutos de linfocitos menores, mayor tiempo } \\
\text { de hospitalización y mayor requerimiento de } \\
\text { UCI. }\end{array}$ & Insomnio $=32 \%$ \\
\hline $\begin{array}{l}\text { Rong Y. et al. } \\
(2020)^{(28)}\end{array}$ & China & $\begin{array}{l}\text { Paciente masculino de } 64 \text { años, hospitalizado } \\
\text { por COVID-19 }\end{array}$ & $\begin{array}{l}\text { Insomnio al inicio de la enfermedad, antes de } \\
\text { presentar las complicaciones neurológicas y } \\
\text { respiratorias. }\end{array}$ & $\begin{array}{l}\text { Insomnio (caso } \\
\text { clínico de un pa- } \\
\text { ciente) }\end{array}$ \\
\hline $\begin{array}{l}\text { Li-yu Lin et al. } \\
(2020)^{(29)}\end{array}$ & China & $\begin{array}{l}\text { Todas las edades. } \\
837 \text { pacientes hospitalizados por COVID- } 19 \text {. } \\
57 \text { pacientes en cuarentena en casa por CO- } \\
\text { VID- } 19 \text {. } \\
660 \text { amigos, colegas y familiares de los ante- } \\
\text { riores sin COVID- } 19 \text {. } \\
4087 \text { sujetos de población general sin CO- } \\
\text { VID-19. }\end{array}$ & $\begin{array}{l}\text { Insomnio durante el pico de la pandemia. } \\
\text { Despertar precoz, despertar durante la noche y } \\
\text { pesadillas. }\end{array}$ & Insomnio $=20,05 \%$ \\
\hline $\begin{array}{l}\text { Hao F. et al. } \\
(2020)^{(26)}\end{array}$ & China & $\begin{array}{l}\text { Mayores de } 18 \text { años. } \\
76 \text { pacientes psiquiátricos (no hospitalizados) } \\
\text { y } 109 \text { controles sanos. }\end{array}$ & $\begin{array}{l}\text { Las personas con antecedentes de trastornos } \\
\text { psiquiátricos presentaron insomnio moderado y } \\
\text { severo comparado con los controles. }\end{array}$ & Insomnio $=27,6 \%$ \\
\hline $\begin{array}{l}\text { Killgore William } \\
\text { et al., }(2020)^{(36)}\end{array}$ & $\begin{array}{l}\text { Estados } \\
\text { Unidos }\end{array}$ & 1013 sujetos sanos entre 18 y 35 años. & $\begin{array}{l}\text { El insomnio se asoció con preocupaciones por } \\
\text { la COVID-19 e ideas suicidas }\end{array}$ & $\begin{array}{c}\text { Insomnio }=56 \% \\
(19,8 \% \text { moderado y } \\
5,2 \% \text { grave }) \text { asocia- } \\
\text { do a ideas suicidas } \\
\quad(p<0,0001)\end{array}$ \\
\hline $\begin{array}{l}\text { Kokou-Kpo- } \\
\text { lou CK et al., } \\
(2020)^{(37)}\end{array}$ & Francia & $\begin{array}{l}556 \text { participantes con edades entre } 18 \text { y } 87 \\
\text { años, de los cuales el 8,6\% estaba infectado de } \\
\text { COVID-19. }\end{array}$ & $\begin{array}{l}\text { El insomnio se asoció a un mayor miedo de } \\
\text { estar infectado con COVID-19 y a un menor } \\
\text { nivel de estudios. }\end{array}$ & Insomnio $=19,1 \%$ \\
\hline $\begin{array}{l}\text { Rossi Ret al. } \\
(2020)^{(38)}\end{array}$ & Italia & 18147 sujetos sanos mayores de 18 años. & $\begin{array}{l}\text { El insomnio se asoció con el sexo femenino } \\
\text { y con vivir preocupado por la infección de la } \\
\text { COVID-19 }\end{array}$ & Insomnio $=7,3 \%$ \\
\hline $\begin{array}{l}\text { Voitsidis P et al., } \\
(2020)^{(39)}\end{array}$ & Grecia & 2363 sujetos sanos mayores de 18 años. & $\begin{array}{l}\text { El insomnio se asoció con el sexo femenino y } \\
\text { vivir en la zona urbana. }\end{array}$ & Insomnio $=37,6 \%$ \\
\hline $\begin{array}{l}\text { Li Y et al., } \\
(2020)^{(40)}\end{array}$ & China & 3637 participantes entre 18 y 76 años. & $\begin{array}{l}\text { Aumentó la prevalencia de insomnio, ansiedad, } \\
\text { depresión y preocupación por COVID- } 19 \text { entre } \\
\text { antes y después del } 21 \text { de enero de } 2020 \text {. }\end{array}$ & $\begin{array}{c}\text { Insomnio antes = } \\
26,2 \% \\
\text { Insomnio después }= \\
33,7 \%\end{array}$ \\
\hline $\begin{array}{l}\text { Zhou S et al. } \\
(2020)^{(41)}\end{array}$ & China & 11835 estudiantes entre 12 y 29 años. & $\begin{array}{l}\text { El insomnio predominó en mujeres. Presencia } \\
\text { de malos hábitos de sueño. }\end{array}$ & Insomnio $=23,2 \%$ \\
\hline $\begin{array}{l}\text { Tan, W. et al. } \\
(2020)^{(42)}\end{array}$ & China & 673 trabajadores sanos (no hospitalizados). & $\begin{array}{l}\text { Los trabajadores volvieron al trabajo con me- } \\
\text { didas de bioseguridad. Las cifras de insomnio } \\
\text { fueron bajas al igual que las de preocupaciones } \\
\text { por la salud. }\end{array}$ & Insomnio $=2,3 \%$ \\
\hline
\end{tabular}

lución de la enfermedad en la comunidad y la cantidad de muertes que se sumaban día a día, lo que les mantenía en constante ansiedad.

El sueño también desempeña un papel importante en la homeostasis del sistema inmunológico, por lo que, el dormir bien y la inmunidad se encuentran directamente relacionados ${ }^{(18,19)}$. La disminución de la calidad de sueño en los pacientes hospitalizados por la COVID-19 parece influir negativamente en la evolución de la enfermedad. Ellos pueden presentar una corta duración de sueño debido a factores como hospitalización en zonas de aislamiento sin la compa- ñía de familiares, miedo, ansiedad, desesperanza, depresión y el malestar causado por la propia enfermedad ${ }^{(20)}$, lo que afectaría su capacidad de respuesta inmunológica permitiendo la activación de los procesos inflamatorios y la liberación de citoquinas ${ }^{(21)}$, y el aumento de la susceptibilidad del paciente a las infecciones respiratorias ${ }^{(22)}$.

En el estudio de Zhang Jiancheng et al. ${ }^{(23)}$, se incluyeron 135 pacientes entre 18 y 80 años que estuvieron hospitalizados en el West District of Wuhan Union Hospital entre enero 25 y marzo 15 de 2020, con diagnóstico de COVID-19 y que presentaban contaje de linfocitos en sangre periférica me- 
nores a $1,1 \times 10^{9} / \mathrm{L}$ en las primeras 24 horas. Los pacientes fueron evaluados con el Richards-Campbell Sleep Questionnaire (RCSQ) y el Pittsburgh Sleep Quality Index (PSQI) y separados en dos grupos según los puntajes obtenidos en las dos escalas, uno de buenos durmientes $(44,4 \%)$ y otro de malos durmientes $(55,6 \%)$. En el grupo de malos durmientes, el 32\% de los pacientes reportó insomnio. Al inicio no existían diferencias en el valor absoluto de linfocitos entre ambos. Cuando se compararon los dos grupos los días 14 y 21 , se pudo apreciar que los malos durmientes presentaron una disminución mayor del valor absoluto de linfocitos $(\mathrm{p}=0,005)$ y $(\mathrm{p}=0,003)$, respectivamente, $y$ un aumento de la relación neutrófilo-linfocito $(\mathrm{p}=0,002)$ y $(\mathrm{p}=0,009)$ respectivamente, situaciones que se han asociado a una peor evolución y muerte en pacientes con la COVID-19 (24,25); además, los pacientes del grupo malos durmientes tuvieron en promedio 8 días más de hospitalización y el 12\% de ellos empeoró y requirieron estar en la unidad de cuidados intensivos (UCI) en comparación con ninguno del grupo de buenos durmientes.

Se ha podido determinar que las personas con antecedentes psiquiátricos han presentado mayor prevalencia de insomnio durante la cuarentena en relación con aquellos sin antecedentes. Hao F et al. ${ }^{(26)}$ compararon 76 pacientes con antecedentes de trastornos de ansiedad o depresión con 109 controles sanos pareados por edad y sexo, y encontraron que más de un cuarto de aquellos con antecedentes presentaban mayores puntuaciones totales en el Insomnia Severity Index (ISI) $(10,1$ vs. 4,$6 ; \mathrm{p}<0,001)$, insomnio moderado $(19,7 \%$ vs. $1,8 \%)$ e insomnio severo ( $7,9 \%$ vs. $0,9 \%)$. Aquellos que informaron una sensación de pobre salud física estuvieron más relacionados con el insomnio $(\mathrm{p}<0,001)$. Se ha descrito que la presencia de antecedentes psiquiátricos puede ser un factor de riesgo para presentar depresión, ansiedad e insomnio durante el aislamiento ${ }^{(27)}$.

Rong Yin et al. ${ }^{(28)}$ describen el caso de un paciente masculino de 64 años, procedente de Wuhan, China, quien presentó inicio de los síntomas para la COVID-19 el 28 de enero de 2020 , fiebre de $38,5^{\circ} \mathrm{C}$ y tos leve. Para el día 30 , el paciente se quejaba de insomnio. Posteriormente la enfermedad evolucionó con complicaciones respiratorias y neurológicas, el paciente sobrevivió y recibió el alta el día 27 de febrero. El insomnio fue un síntoma inicial antes de presentar alteraciones de conciencia, letargo, irritabilidad, reflejo de Babinski, Chaddock y Brudzinski.

El estudio de Li-yu Lin et al. ${ }^{(29)}$ se realizó en China mediante una encuesta por internet; incluyó a 5641 sujetos de todas las edades, divididos en varios grupos: pacientes que estuvieron hospitalizados por la COVID-19 y trabajadores de la salud de primera línea; personas con el diagnóstico o síntomas pero que cumplieron la cuarentena en casa; amigos, colegas y familiares de los anteriores; y población general que cumplen las estrategias de prevención. La prevalencia de in- somnio durante el mes de febrero, considerado el pico de la pandemia, fue de $20 \%$ con el ISI para el total de la muestra. Las alteraciones fueron despertar precoz, despertar frecuente durante la noche y pesadillas. Las razones del trastorno de sueño fueron las preocupaciones por la enfermedad y la mala higiene del sueño, ya que, debido a la cuarentena pasaban más tiempo en cama viendo noticias en la televisión o utilizando sus teléfonos para jugar o revisar las redes sociales, lo cual alteraba su ritmo biológico de sueño. Los sujetos de los grupos 2 y 4 retrasaron su hora de ir a la cama 60 minutos o más, debido a que no tenían que trabajar y otros realizaban siestas durante el día. A los participantes se les pidió recordar cómo había sido su sueño durante el último trimestre de 2019, antes de la pandemia, e informaron que solo el 14,6\% había presentado insomnio durante esos meses.

\section{INSOMNIO EN PERSONAS SOMETIDAS A AISLAMIENTO O CUARENTENA DEBIDO A LA COVID-19}

Se entiende por arousal una rápida desincronización de la actividad bioeléctrica cerebral, acompañada o no de un incremento en la actividad muscular y vegetativa. El término arousal es sinónimo de "activación cerebral» y cuando es atribuido al insomnio se denomina hiperarousal. Suele ocurrir durante el sueño como consecuencia de estímulos exógenos, endógenos o de un elevado nivel de activación interna corporal (psíquica o somática). El término hiperarousal se entiende también como situación mantenida de vigilancia que puede estar ocurriendo durante el día.

El hiperarousal del insomnio sería consecuencia del alertamiento emocional provocado por la internalización de la emoción con un incremento mantenido de diversos fenómenos vegetativos (temperatura, frecuencia cardíaca, tasa metabólica, etc.) durante el día y una dificultad para iniciar el sueño en la noche. Así, se puede concluir que las consecuencias del hiperarousal diurno serían la dificultad de iniciar y mantener el sueño ${ }^{(30)}$.

El confinamiento en casa y el temor a contraer la COVID-19, sumados a los problemas económicos, distanciamiento social, restricciones para viajar, cambios en el estilo de vida y medidas para impedir la propagación de la enfermedad entre la población, como uso de guantes, tapabocas y lentes protectores, son factores generadores de estrés que sin duda pueden actuar como generadores de hiperarousal y desencadenantes del insomnio ${ }^{(31-33)}$. Otro factor para tomar en consideración son los cambios de hábitos del sueño y de la vigilia durante el confinamiento, cuando las personas se acuestan más tarde y gastan más tiempo en cama sin dormir, dejando de lado la higiene del sueño ${ }^{(34)}$.

El insomnio puede ser primario o secundario (comórbido) a situaciones de estrés ${ }^{(12)}$. Las personas que experimen- 
tan mayores niveles de ansiedad y preocupación tienden a presentar a su vez menor calidad de sueño, más síntomas de insomnio y mayor tiempo de latencia de sueño ${ }^{(35)}$, algo que podría esperarse durante la cuarentena por COVID-19.

Un estudio llevado a cabo en los Estados Unidos ha revelado que las preocupaciones por la COVID-19 asociadas con el insomnio pueden desencadenar ideas suicidas en algunas personas. Killgore William et al. ${ }^{(36)}$ aplicaron una encuesta a 1013 sujetos sanos entre 18 y 35 años, para evaluar insomnio con el ISI, suicidio con el Patient Health Questionnaire-9 (PHQ-9) y preocupación por la COVID-19 mediante la pandemic worry scale. Encontraron que el $56 \%$ de los participantes reportaron insomnio ( $19,8 \%$ moderado y $5,2 \%$ grave), lo cual se asoció a preocupaciones por la COVID-19 ( $\mathrm{p}<0,001)$ $y$ a ideas suicidas $(\mathrm{p}<0,001)$. Concluyen que una intervención terapéutica para mejorar el insomnio desde el inicio podría disminuir las ideas suicidas de manera temprana.

Resultados similares se han encontrado en países europeos. Kokou-Kpolou CK et al. ${ }^{(37)}$ realizaron un estudio en Francia, entre el 3 y 16 de mayo, mediante una entrevista en línea para determinar la prevalencia de insomnio y los factores asociados en población general francesa. Incluyeron 556 participantes entre 18 y 87 años, de los cuales el 8,6\% tenían la COVID-19. El 19,1\% presentó insomnio que no se relacionó con el género; sin embargo, las personas con educación universitaria presentaron menos insomnio que aquellos con estudios de primaria y secundaria (OR 2,59; IC 95\%: 1,34 a 5,02). Dentro de los factores de riesgo para el insomnio se encontraron, altos niveles de preocupación acerca de la COVID-19 (OR 1,39; IC 95\%: 1,09 a 1,78) y antecedentes de enfermedades mentales (OR 1,22; IC 95\%: 1,12 a 1,33).

Rodolfo Rossi et al. ${ }^{(38)}$ aplicaron un cuestionario en línea entre el 27 de marzo y 6 de abril de 2020; durante el pico de la pandemia, a 18147 ciudadanos italianos sin la enfermedad y mayores de 18 años. Se valoró insomnio (ISI), la percepción de estrés (PSS), depresión (PHQ-9), ansiedad (Generalized Anxiety Disorder scale) (GAD-7) y trastorno de estrés postraumático (GPS-PTSS). Solo un 7,3\% de los participantes refirió síntomas severos de insomnio y un 20,8\% ansiedad grave. Los factores que se asociaron al insomnio fueron el sexo femenino (OR 1,50; IC 95\%: 1,26 a 1,78); vivir en el sur de Italia que era la zona más afectada en ese momento (OR 1,41; IC 95\%: 1,24 a 1,62); sentirse estresado debido a la COVID-19 (OR 1,58; IC 95\%: 1,40 a 1,79); haber dejado de trabajar debido a la COVID-19 (OR 1,22; IC 95\%: 1,03 a $1,46)$ y que un ser querido haya fallecido por la COVID-19 (OR 1,74; IC 95\%: 1,18 a 2,54). Ellos consideran que la baja prevalencia de insomnio en comparación con la población china puede deberse a factores culturales y sociales.

Voitsidis P et al. ${ }^{(39)}$ llevaron a cabo un estudio en Grecia tres semanas después de implementada la cuarentena, entre el 10 y el 13 de abril de 2020, para determinar el impacto de la COVID-19 en el sueño. Entrevistaron vía web un total de
2363 sujetos sanos mayores de 18 años, quienes completaron la Athens Insomnia Scale (AIS). 37,6\% de los participantes puntearon para insomnio. Los factores asociados fueron el sexo femenino $(p=0,001)$ y vivir en la ciudad $(p<0,001)$. Este es otro estudio donde las preocupaciones por la COVID-19 y la sensación de soledad también se asociaron al insomnio. Además de los factores mencionados en otros trabajos, ellos consideraron que la poca exposición al sol debido al aislamiento puede ser un factor que influye en el ciclo sueño vigilia.

También se ha evaluado el insomnio en diversos momentos de la pandemia. Li Yun et al. ${ }^{(40)}$ aplicaron el ISI en línea a 3637 participantes entre 18 y 76 años de 31 provincias en China. Todos los participantes llenaron una encuesta retrospectiva para evaluar el insomnio entre los días 6 y 20 de enero de 2020. Se utilizó la misma encuesta para evaluar el insomnio otra vez a partir del 21 de enero, luego de confirmarse la transmisión persona a persona de la enfermedad. También se aplicó la GAD-7 y el PHQ-9. La prevalencia de insomnio aumentó de 26,2\% a 33,7\% ( $\mathrm{p}<0,001)$. También se encontraron cambios en la hora de irse a la cama, la hora de levantarse y la prevalencia de ansiedad, depresión y preocupaciones por infectarse. Además del miedo a la enfermedad, los cambios en el estilo de vida pudieron haber influido en el aumento de la prevalencia de insomnio.

Shuang-Jiang Zhou et al. ${ }^{(41)}$ realizó un estudio en población joven, incluyeron a 11835 estudiantes entre 12 y 29 años de 21 provincias de China, a quienes se les aplicó un cuestionario en línea entre el 8 y 15 de marzo 2020. Los síntomas de insomnio se evaluaron con el PSQI, también se aplicó el PHQ-9 y la GAD-7. Las mujeres presentaron más síntomas de insomnio que los hombres. El 23,2\% de la muestra experimentó síntomas de insomnio, con 18,0\%, 25,3\% y 25,7\% en estudiantes de educación media, educación secundaria y superior, respectivamente. Los adolescentes con menos horas de clase tuvieron una tendencia a malos hábitos de sueño, como acostarse y levantarse más tarde, usar el teléfono, jugar y hacer compras en línea, lo que lleva a mayor latencia de sueño y menor tiempo de sueño. Aun cuando la edad joven se ha asociado a insomnio, este estudio muestra una prevalencia similar en los participantes adultos.

Salir del confinamiento y volver al trabajo cumpliendo las medidas higiénicas de seguridad, como uso de mascarillas, lavado frecuente de manos y distanciamiento social podría mejorar el insomnio de las personas que han estado en cuarentena y aislamiento en casa. Los días 24 y 25 de febrero de 2020, Tan W et al. ${ }^{(42)}$ hicieron una encuesta en línea a 1323 trabajadores de varias compañías de Chongqing, China, que regresaban al trabajo durante la cuarentena, con estrictas medidas de seguridad. Se incluyeron 673 sujetos tanto de la fuerza laboral como ejecutivos mayores de 18 años y sin antecedentes de enfermedades mentales a quienes se aplicó el ISI. Solo un 2,3\% de ellos presentaba insomnio (modera- 
do $1,9 \%$ y severo $0,4 \%$ ), muy similar a los participantes que manifestaron estar preocupados por su salud (preocupados $2,5 \%$ y muy preocupados $2,4 \%$ ).

\section{CONCLUSIONES}

Diferentes estudios han mostrado la influencia negativa del confinamiento sobre el sueño. En muchos casos la ansiedad y el estrés han traído como consecuencia insomnio y en otros han sido los cambios en el estilo de vida, o el uso de dispositivos electrónicos a la hora de ir a dormir. Sin embargo, esta situación causada por la pandemia no tiene precedentes, y los instrumentos que se han utilizado para valorar los trastornos del sueño no están diseñados para medir el insomnio en estas condiciones. Aun así, esto no ha impedido que los investigadores se hayan esforzado en tratar de medir las consecuencias negativas sobre el sueño, llegando a primeras conclusiones de que el insomnio parece estar asociado con el sexo femenino y a una edad joven, a personas con antecedentes psiquiátricos y a la forma como se perciben el estrés por miedo al contagio. Asimismo, se ha determinado que dormir mal puede alterar el sistema inmunológico haciendo a los pacientes con la COVID-19 más susceptibles a las complicaciones respiratorias.

Desde el punto de vista de salud pública es importante destacar que mantener una buena higiene del sueño debería repercutir positivamente en disminuir las complicaciones generadas por enfermedades crónicas, como hipertensión o diabetes, entre otras, permitiendo a los pacientes una mayor capacidad de afrontamiento a la enfermedad del COVID-19, o por lo menos evitando empeoramiento en estas enfermedades de base, las cuales han sido asociadas como factores de riesgo para COVID-19, además de que ya de por sí, el solo aumento de la prevalencia de insomnio en la población general viene a representar un nuevo problema en salud pública.

\section{RECOMENDACIONES}

Durante la cuarentena se deben tener en consideración los aspectos básicos de la higiene del sueño, como levantarse siempre a la misma hora y no utilizar aparatos electrónicos dos horas antes de acostarse, entre otros, así como, tratar de recibir la luz del sol durante el día, y practicar rutinas de relajación en casa, al igual que no empaparse a diario con el conteo de personas infectadas o de muertes anunciadas en la televisión o en las redes sociales ${ }^{(43)}$. Considerando que Latinoamérica ha sido una de las regiones con una gran cifra de contagios y muertes debido en gran parte a que no se contaba con la preparación necesaria en políticas de salud para afrontar la pandemia, es indispensable promover campañas de prevención, dentro de las posibilidades socioeconómicas de cada país, que puedan tranquilizar a los ciudadanos, lo cual evitaría la propagación del temor y la ansiedad, a fin de que estos aspectos no influyan en su calidad de sueño.

Contribuciones de autoría: Todos los autores han colaborado en la búsqueda de los artículos científicos, la revisión crítica del artículo y la aprobación de la versión final

Financiamiento: Autofinanciado.

Conflictos de interés: Los autores declaran no tener conflictos de interés.

\section{REFERENCIAS BIBLIOGRÁFICAS}

1. Del Rio C, Malani PN. 2019 Novel Coronavirus-Important Information for Clinicians. JAMA. 2020; 323(11):1039-1040. doi: 10.1001/ jama.2020.1490.

2. Cheng ZJ, Shan J. 2019 Novel coronavirus: where we are and what we know. Infection.Apr; 48(2):155-63. doi: 10.1007/s15010-02001401-y. Epub 2020 Feb 18.

3. Huang C, Wang Y, Li X, Ren L, Zhao J, Hu Y, et al. Clinical features of patients infected with 2019 novel coronavirus in Wuhan, China. Lancet. 2020;395(10223):497-506. doi: https://doi.org/10.1016/S01406736(20)30183-5.

4. Backer JA, Klinkenberg D, Wallinga J. Incubation period of 2019 novel coronavirus (2019-nCoV) infections among travelers from Wuhan, China, 20-28. 2020; Euro Surveill. 2020 Feb 6; 25(5): 2000062 doi: 10.2807/1560-7917.ES.2020.25.5.2000062.

5. Chen N, Zhou M, Dong X, Qu J, Gong F, Han Y, et al. Epidemiological and clinical characteristics of 99 cases of 2019 novel coronavirus pneumonia in Wuhan, China: a descriptive study. Lancet. 2020;395(10223):507-13. doi: https://doi.org/10.1016/S01406736(20)30211-7.

6. Centers for Disease Control and Prevention (CDC). Quarantine and Isolation [Internet]. 2017 [cited 2020 Jul 05]. Available from: https:// www.cdc.gov/quarantine/index.html.
7. Jeong H, Yim H, Song Y, Ki M, Min J, Cho J, et al. Mental health status of people isolated due to Middle East Respiratory Syndrome. Epidemiol Health. 2016;38:e2016048. doi: 10.4178/epih.e2016048.

8. Hossain MM, Sultana A, Purohit N. Mental health outcomes of quarantine and isolation for infection prevention: A systematic umbrella review of the global evidence. Epidemiol health. 2020:e2020038. doi: 10.4178/epih.e2020038.

9. Krystal AD. Psychiatric disorders and sleep. Neurologic clinics. 2012;30(4):1389-413. doi: 10.1016/j.ncl.2012.08.018.

10. Medina Ortiz Óscar, Sánchez Mora Nora, Conejo Galindo Javier, Fraguas Herráez David, Arango López Celso. Alteraciones del sueño en los trastornos psiquiátricos. Rev Colomb Psiquiatr. [Internet]. 2007 Dec [cited 2020 Oct 12]; 36(4): 701-717. Available from: http://www.scielo.org.co/scielo.php?script=sci_arttext\&pi$\mathrm{d}=$ S0034-74502007000400009\&lng=en.

11. Yu Wang, Li-Yun Zhu, Yu-Fen Ma, Hai-Xin Bo, Hai-Bo Deng, Jing Cao, et al. Association of insomnia disorder with sociodemographic factors and poor mental health in COVID-19 inpatients in China. Sleep Med. 2020: In Press https://doi.org/10.1016/j.sleep.2020.06.011.

12. American Academy of Sleep Medicine. International classification of sleep disorders. $3^{\mathrm{a}}$ ed Darien (IL). 2014. 
13. Morin CM, LeBlanc M, Daley M, Gregoire JP, Merette C. Epidemiology of insomnia: prevalence, self-help treatments, consultations, and determinants of help-seeking behaviors. Sleep Med. 2006;7(2):123-30. doi: 10.1016/j.sleep.2005.08.008.

14. Riemann D, Baglioni C, Bassetti C, Bjorvatn B, Dolenc Groselj L, Ellis JG, et al. European guideline for the diagnosis and treatment of insomnia. J Sleep Res. 2017;26(6):675-700. doi: 10.1111/jsr.12594.

15. Ohayon MM. Epidemiology of insomnia: what we know and what we still need to learn. Sleep Med Rev. 2002; 6:97-111. doi: 10.1053/ smrv.2002.0186.

16. Morán San Juan L, Mayoral Sánchez J. El relato del miedo en las noticias de televisión. Análisis de los informativos de TVE 1 y Telecinco [Internet]. 2017 [cited 2020 Jul 07]. Available from: https://eprints. ucm.es/47289/1/T39938.pdf. Madrid: Universidad Complutense de Madrid; 2018. Tesis Doctoral.

17. Wang Y, Zhu L-Y, Ma Y-F, Bo H-X, Deng H-B, Cao J, et al. Association of insomnia disorder with sociodemographic factors and poor mental health in COVID-19 inpatients in China. Sleep Med. 2020; In Press available in https://doi.org/10.1016/j.sleep.2020.06.011.

18. Besedovsky L, Lange T, Haack M. The Sleep-Immune Crosstalk in Health and Disease. Physiol Rev. 2019;99(3):1325-80. doi: 10.1152/ physrev.00010.2018.

19. Haspel JA, Anafi R, Brown MK, Cermakian N, Depner C, Desplats $P$, et al. Perfect timing: circadian rhythms, sleep, and immunity - an NIH workshop summary. JCI Insight. 2020 Jan 16;5(1):e131487. doi: 10.1172/jci.insight.131487.

20. Guo Q, Zheng Y, Shi J, Wang J, Li G, Li C, et al. Immediate psychological distress in quarantined patients with COVID-19 and its association with peripheral inflammation: A mixed-method study. Brain Behav Immun. 2020 Aug; 88: 17-27. Published online 2020 May 19. doi: 10.1016/j.bbi.2020.05.038.

21. Irwin MR. Why sleep is important for health: a psychoneuroimmunology perspective. Annu Rev Psychol. 2015; 66:143-72. doi: 10.1146/ annurev-psych-010213-115205.

22. Loef B, van Baarle D, van der Beek AJ, Sanders EAM, Bruijning-Verhagen P, Proper KI. Shift Work and Respiratory Infections in Health-Care Workers. Am J Epidemiol. 2019;188(3):509-17. DOI: 10.1093/aje/ kwy258.

23. Zhang J, Xu D, Xie B, Zhang Y, Huang H, Liu H, et al. Poor-sleep is associated with slow recovery from lymphopenia and an increased need for ICU care in hospitalized patients with COVID-19: A retrospective cohort study. Brain Behav Immun. 2020 Aug; 88: 50-58. Published online 2020 Jun 6. doi: 10.1016/j.bbi.2020.05.075.

24. Zhou F, Yu T, Du R, Fan G, Liu Y, Liu Z, et al. Clinical course and risk factors for mortality of adult inpatients with COVID-19 in Wuhan, China: a retrospective cohort study. Lancet. 2020;395(10229):1054-62. doi: https://doi.org/10.1016/S0140-6736(20)30566-3.

25. Lagunas-Rangel FA. Neutrophil-to-lymphocyte ratio and lymphocyte-to-C-reactive protein ratio in patients with severe coronavirus disease 2019 (COVID-19): A meta-analysis. J Med Virol. First published: 03 April 2020 https://doi.org/10.1002/jmv.25819.

26. Hao F, Tan W, Jiang L, Zhang L, Zhao X, Zou Y, et al. Do psychiatric patients experience more psychiatric symptoms during COVID-19 pandemic and lockdown? A case-control study with service and research implications for immunopsychiatry. Brain Behav Immun. 2020; 87:100-6. Published online 2020 Apr 27. doi: 10.1016/j.bbi.2020.04.069

27. Wang C, Pan R, Wan X, Tan Y, Xu L, Ho CS, et al. Immediate Psychological Responses and Associated Factors during the Initial Stage of the 2019 Coronavirus Disease (COVID-19) Epidemic among the General Population in China. Int J Environ Res Public Health. 2020;17(5):1720. doi: 10.3390/ijerph17051729.

28. Rong Y, Wei F, Tonghui W, Gang C, Tao W, Dongrui C, et al. Concomitant neurological symptoms observed in a patient diagnosed with coronavirus disease 2019. J Med Virol. 2020 Apr 15; In Press. Available in https://doi.org/10.1002/jmv.25888.

29. Lin LY, Wang J, Ou-Yang XY, Miao Q, Chen R, Liang FX, et al. The immediate impact of the 2019 novel coronavirus (COVID-19) outbreak on subjective sleep status. Sleep Med. 2020. In press. doi: 10.1016/j. sleep.2020.05.018.

30. Espinar-Sierra J. Arousal and its influence on vigilance. Rev Neurol. 1999;28(6):555-9. doi: https://doi.org/10.33588/rn.2806.98457.

31. Healey ES, Kales A, Monroe LJ, Bixler EO, Chamberlin K, Soldatos CR. Onset of insomnia: role of life-stress events. Psychosom Med. 1981;43(5):439-51. doi: 10.1097/00006842-198110000-00007.

32. Drake CL, Pillai V, Roth T. Stress and sleep reactivity: a prospective investigation of the stress-diathesis model of insomnia. Sleep. 2014;37(8):1295-304. doi: 10.5665/sleep.3916.

33. Marazziti D, Stahl SM. The relevance of COVID-19 pandemic to psychiatry. World psychiatry: official journal of the World Psychiatric Association. 2020;19(2):261. doi: 10.1002/wps.20764.

34. Mônico-Neto M, Thomatieli dos Santos RV, Moreira Antunes HK. The world war against the COVID-19 outbreak: don't forget to sleep! J Clin Sleep Med. 2020; In Press. Available in https://doi.org/10.5664/ jcsm.8502.

35. Horvath A, Montana X, Lanquart JP, Hubain P, Szucs A, Linkowski P, et al. Effects of state and trait anxiety on sleep structure: A polysomnographic study in 1083 subjects. Psychiatry Res. 2016; 244:279-83. doi: 10.1016/j.psychres.2016.03.001.

36. Killgore WDS, Cloonan SA, Taylor EC, Fernandez F, Grandner MA, Dailey NS. Suicidal ideation during the COVID-19 pandemic: The role of insomnia. Psychiatry Res. 2020; 290:113134. doi: 10.1016/j. psychres.2020.113134

37. Kokou-Kpolou CK, Megalakaki O, Laimou D, Kousouri M. Insomnia during COVID-19 pandemic and lockdown: Prevalence, severity, and associated risk factors in French population. Psychiatry Res. 2020; 290:113128. doi: 10.1016/j.psychres.2020.113128.

38. Rossi R, Socci V, Talevi D, Mensi S, Niolu C, Pacitti F, et al. COVID-19 pandemic and lockdown 1 measures impact on mental 2 health among the general population in Italy. An N=18147 web-based 3 survey. medRxiv 2020040920057802; In Press Available in: https:// doiorg/101101/2020040920057802.

39. Voitsidis P, Gliatas I, Bairachtari V, Papadopoulou K, Papageorgiou G, Parlapani E, et al. Insomnia during the COVID-19 pandemic in a Greek population. Psychiatry Res. 2020 Jul; 289: 113076. Published online 2020 May 12. doi: 10.1016/j.psychres.2020.113076.

40. Li Y, Qin Q, Sun Q, Sanford LD, Vgontzas AN, Tang X. Insomnia and psychological reactions during the COVID-19 outbreak in China. J Clin Sleep Med: JCSM : official publication of the American Academy of Sleep Medicine. 2020. J Clin Sleep Med 2020 Aug 15;16(8):14171418. doi: $10.5664 /$ jcsm. 8524 .

41. Zhou S-J, Wang L-L, Yang R, Yang X-J, Zhang L-G, Guo Z-C, et al. Sleep problems among Chinese adolescents and young adults during the coronavirus-2019 pandemic. Sleep Med. 2020; In Press. Available in https://doi.org/10.1016/j.sleep.2020.06.001.

42. Tan W, Hao F, McIntyre RS, Jiang L, Jiang X, Zhang L, et al. Is returning to work during the COVID-19 pandemic stressful? A study on immediate mental health status and psychoneuroimmunity prevention measures of Chinese workforce. Brain Behav Immun. 2020 Jul; 87: 84-92. Published online 2020 Apr 23. doi: 10.1016/j.bbi.2020.04.055.

43. Altena E, Baglioni C, Espie CA, Ellis J, Gavriloff D, Holzinger B, et al. Dealing with sleep problems during home confinement due to the COVID-19 outbreak: Practical recommendations from a task force of the European CBT-I Academy. J Sleep Res. 2020:e13052. First published: 04 April 2020. https://doi.org/10.1111/jsr.13052. 